\title{
Accelerated Canine Retraction using Two Distraction Methods - A Case Report
}

\author{
Neetu Dabla ${ }^{1}$, Arjun Vedvyas ${ }^{2}$, Narayana Prasad $^{3}$ \\ ${ }^{1}$ Department of Orthodontics, Eklavya Dental College, Kotputli, Jaipur, Rajasthan, India, ${ }^{2}$ Private Practitioner, Indirapuram, \\ Ghaziabad, Uttar Pradesh, India, ${ }^{3}$ Department of Orthodontics, Seema Dental College, Rishikesh, Uttarakhand, India
}

Email for correspondence: neetudabla@gmail.com

\begin{abstract}
Accelerating rate of orthodontic treatment has gained significant popularity due to the increased demand from orthodontic patients to reduce the time taken for orthodontic mechanotherapy. Rapid canine retraction employing various distraction methods has been employed for severe crowding, Angle's Class II Division 1 malocclusions, bidentoalveolar protrusion, root shortening, and malformation, as well as in patients presenting with periodontal problems. Various distraction methods are available for canine retraction. The present case report demonstrates the effective use of two different distraction methods to accelerate canine retraction, and subsequent reduction in overall orthodontic treatment time.
\end{abstract}

Key words: Canine retraction, corticotomy, dentoalveolar distraction, tooth movement acceleration

\section{INTRODUCTION}

Distraction osteogenesis is a method of inducing new bone formation by applying mechanical strains on the preexisting bone. The formation of new bone is achieved through stretching of the callus in the osteotomy or corticotomy gap with distraction devices. The formation of the new bone in the osteotomy or corticotomy site with a width of approximately $1 \mathrm{~mm}$ per day can be achieved by this method. ${ }^{[1]}$

Distraction osteogenesis was used as early as 1905 by Codivilla ${ }^{[2]}$ for femoral lengthening and was later popularized by the clinical and research studies of Ilizarov ${ }^{[3]}$ a Russian orthopedic surgeon in 1950's. ${ }^{[4-6]}$ McCarthy et al. were the first to apply distraction osteogenesis to the craniofacial skeleton in 1990's in children having congenital craniofacial anomalies. ${ }^{[7-9]}$ Since then, it has been applied to various bones of the craniofacial skeleton.

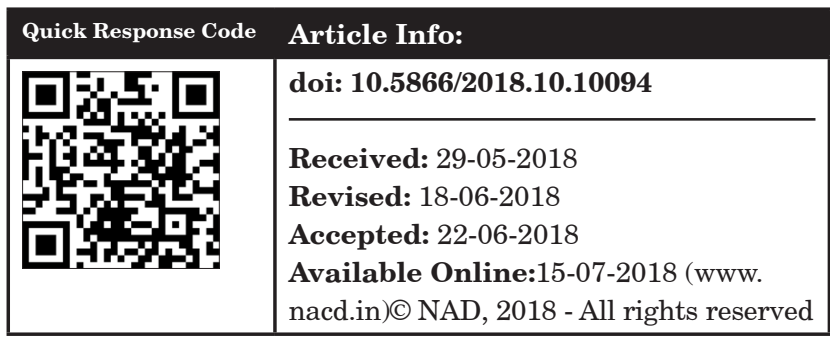

In 1998, Liou and Huang introduced the concept of distraction osteogenesis in tooth movement and termed it as periodontal ligament distraction osteogenesis. $^{[10]}$ In 2002, Iseri et al. introduced another technique for rapid canine retraction (RCR). In this approach, known as a dentoalveolar distraction (DAD), the segment that contains the canine is transported as a bone block. ${ }^{[11]}$ Regardless of the technique, RCR has proven well suited for the following clinical situations: Severe crowding, Angle's Class II Division 1 malocclusions, bidentoalveolar protrusion, root shortening, and malformation, as well as in patients presenting with periodontal problems. RCR has certain definite advantages over conventional orthodontic treatment such as a significant reduction in treatment time from 6 to 9 months, elimination of intra- or extraoral anchorage during the procedure, which ensures nearly complete anchorage preservation.

The present case report demonstrates the effective use of two different distraction methods to accelerate canine retraction and subsequent reduction in overall orthodontic treatment time.

\section{CASE REPORT}

A 20-year-old female patient reported to the department of orthodontics and dentofacial orthopaedics with the chief complaint of "forwardly placed teeth in upper front region." On clinical 
examination, the patient had proportional facial thirds and a convex profile [Figure 1]. Intraoral examination revealed that patient had Angle's Class II Division 1 malocclusion with overjet of $6 \mathrm{~mm}$, an overbite of $30 \%$ [Figure 2].

The treatment plan consisted of initial leveling and aligning followed by extraction of maxillary first premolars and mandibular premolars and RCR followed by fixed appliance orthodontic treatment, with no use of extraoral or intraoral anchorage appliances. The treatment objectives were to correct the inclination of incisors in both the arches, correct the midline deviation, achieve a normal occlusion with ideal overjet and overbite, reduce the protrusion of the lower lip, and improve the facial profile.

\section{Surgical procedure \\ DAD}

The flap design was, a crevicular incisions were made extending from the mesial interdental papilla of the first molar to the mesial interdental papilla of the lateral incisor on the corresponding side. In addition, a vertical releasing incision beginning mesial to the distal interdental papilla of the lateral incisor at the vestibule was made. A mucoperiosteal flap was elevated $6 \mathrm{~mm}$ above the canine and first premolar to allow for the planned osteotomy for both the procedures. A horizontal mucosal incision was made under local anesthesia parallel to the gingival margin of the canine and the premolar beyond the depth of the vestibule. ${ }^{[11]}$ Cortical holes were made in the alveolar bone with a small and round carbide bur from the canine to the second premolar, curving apically to pass $3-5 \mathrm{~mm}$ from the apex [Figure 3] and fine osteotomes were advanced in the coronal direction. The first premolar was extracted and the buccal bone removed between the outlined bone cut at the distal canine region anteriorly and the second premolar posteriorly. The buccal and apical bone through the extraction socket and the possible bony interferences at the buccal aspect that might be encountered during the distraction process were eliminated or smoothed between the canine and the second premolar, preserving palatal/lingual cortical shelves. Osteotomes along the anterior aspect of the canine were used to split the surrounding bone around its root from the palatal or lingual cortex and neighboring teeth. The transport dentoalveolar segment that includes the canine also includes the buccal cortex and the underlying spongy bone that envelopes the canine root, leaving an intact lingual or palatal cortical plate, and the bone around the apex of the canine. The incision was closed with absorbable sutures, and an antibiotic and a nonsteroidal antiinflammatory drug were prescribed for 5 days.

\section{Periodontal ligament distraction}

After first premolar extraction, the interseptal bone distal to the canine was undermined with a bone bur, grooving vertically inside the extraction socket, along the buccal and lingual sides, and extending obliquely toward the base of the interseptal bone to weaken its resistance. ${ }^{[10]}$ The interseptal bone was not cut through mesiodistally toward the canine [Figure 4]. The depth of the undermining grooves was dependent on the thickness of the interseptal bone, as revealed on the periapical films.

\section{Treatment progress}

A custom-made intraoral distractor was placed after the surgical procedure. The distraction
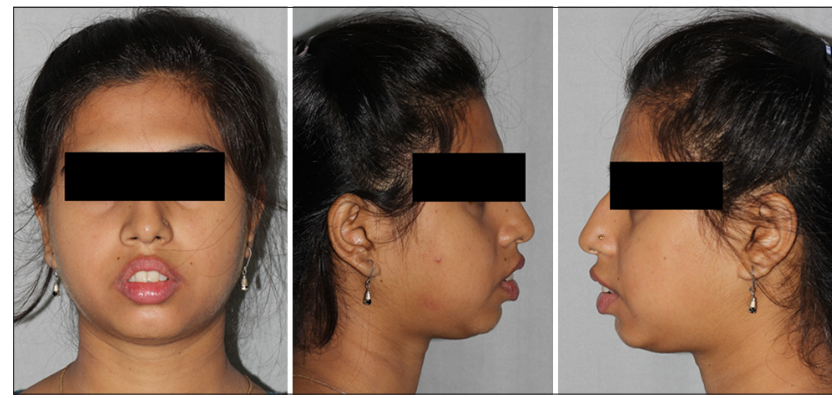

Figure 1: Extraoral picture reveals a patient having proportional facial thirds with convex profile
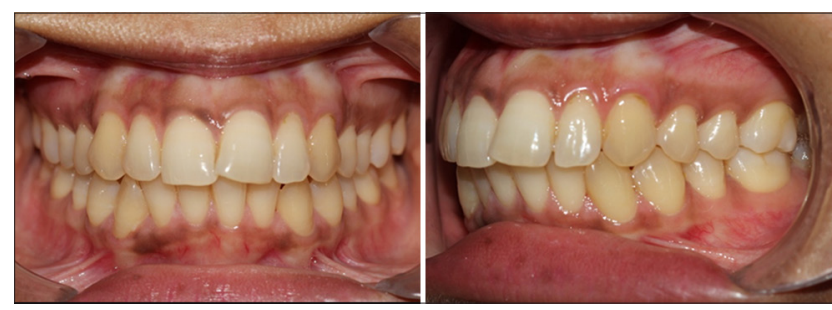

Figure 2: Intraoral pictures showing an overjet of $6 \mathrm{~mm}$ and about $30 \%$ overbite
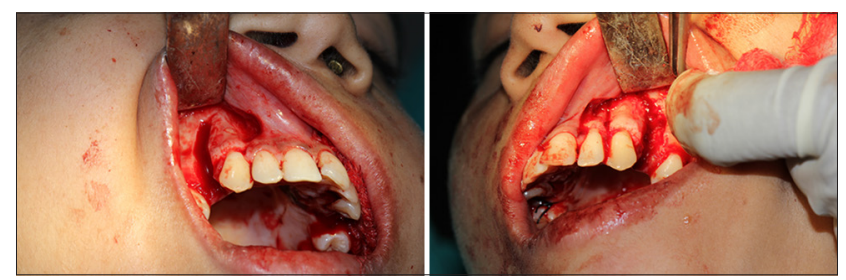

Figure 3: Cortical holes were made in alveolar bone from canine to second premolar, curving apically to pass $3-5 \mathrm{~mm}$ from the apex 
procedure was initiated immediately on the side of periodontal distraction, and after 3 days of latency period, it was initiated on the side of DAD. ${ }^{[10,11]}$ The distraction device was activated twice a day at a rate of $0.4 \mathrm{~mm} /$ day. $^{[10]}$ After the completion of the distraction procedure, the treatment was continued with conventional fixed appliances.

The canines were fully retracted in 15 days on the periodontal side and 13 days on the DAD side [Figure 5]. After completion of distraction, the distractor was removed, and brackets were bonded. The canine and the second premolar were then ligated together. Periapical radiographs of the canines and first molars and lateral cephalogram were obtained before and after the distraction procedure to measure the amount of retraction achieved during distraction. ${ }^{[1,11]}$ No anchorage loss was noticed

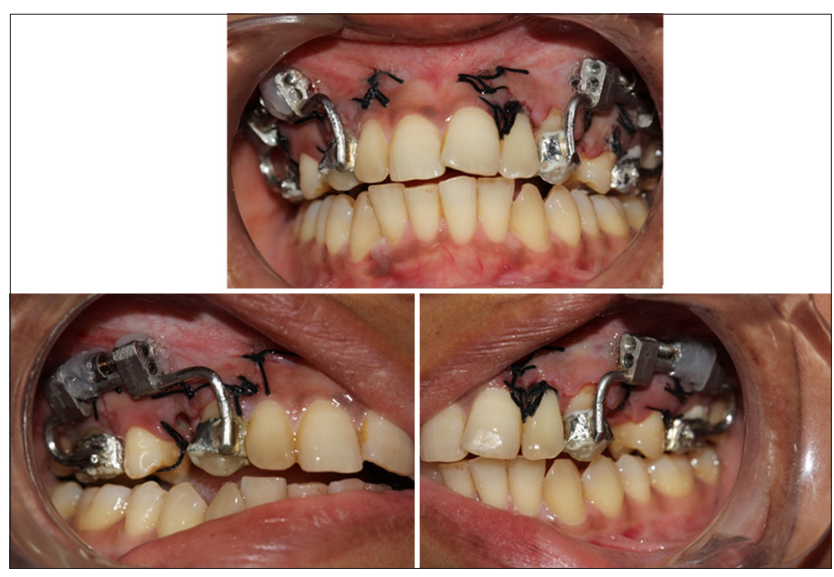

Figure 4: Periodontal ligament distraction on one side and dentoalveolar distraction on other side initiated as per individual surgical procedure on each side

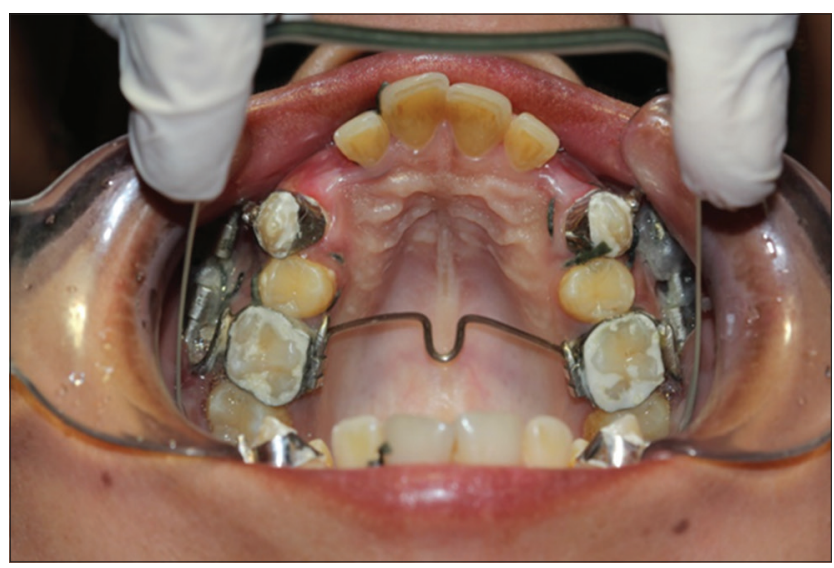

Figure 5: Distraction completed and canines were fully retracted in 15 days on the periodontal side and 13 days on the dentoalveolar distraction side on both the sides. The distal displacement of the canines was $7.3 \mathrm{~mm}$ on the periodontal distraction side at the rate of $0.48 \mathrm{~mm} /$ day and $7.55 \mathrm{~mm}$ on the side of DAD at the rate of $0.58 \mathrm{~mm} /$ day. An angulation change of 6 degrees was observed in canine inclination. Patient reported minimal to moderate discomfort, especially during the first 2 days after surgery, and mild edema was observed which got resolved in 2 days.

\section{DISCUSSION}

Orthodontic tooth movement is a process whereby the application of a force induces bone resorption on the pressure side and bone apposition on the tension side. ${ }^{[12,13]}$ The rate of biologic tooth movement with optimum mechanical force is approximately $1-1.5 \mathrm{~mm}$ in $4-5$ weeks. ${ }^{[14]}$ Hence, in maximum anchorage premolar extraction cases, canine distalization usually takes 6-9 months, contributing to an overall treatment time of 1.5-2 years. Many attempts have been made to shorten the time for orthodontic tooth movement. Liou and Huang reported a RCR technique involving distraction of the PDL after extraction of the first premolars. ${ }^{[10]}$ Iseri et al. described and clinically used a new technique for rapid retraction of the canines known as "DAD."[11]

The term physiologic tooth movement denotes primarily, slight tipping of the tooth in its socket and secondarily, the changes in tooth position that occurs during and after tooth eruption. ${ }^{[15,16]}$ It has been assumed that the application of force during orthodontic treatment leads to hyalinization caused partly by anatomic and partly by mechanical factors. ${ }^{[17]}$ The hyalinization period usually past 2 or 3 weeks and tooth movement continues at a rate of $1-1.5 \mathrm{~mm}$ in $4-5$ weeks. ${ }^{[14]}$ On the contrary with the custom-made, rigid, and tooth-borne distraction device used in this case, the canines were retracted at a rate of $0.5 \mathrm{~mm}$ per day and moved into the socket of the extracted first premolars in compliance with distraction osteogenesis principles. Although every attempt was made to achieve bodily movement of the canines with distraction osteogenesis, some amount of tipping of the canines was observed. Therefore, the distal displacement of the canines was mainly a combination of tipping and translation. Complete retraction of the canines was achieved, and the anchorage teeth were able to withstand the retraction forces with minimal anchorage loss.

There was no clinical and radiographic evidence of complications such as-root fracture, root 
resorption, ankylosis, and soft tissue dehiscence was observed. It has been reported that there occurs an association between the duration of the applied force and increased root resorption. The best way to minimize root resorption is to complete the tooth movement in a short time. Root resorption begins 2-3 weeks after the orthodontic force is applied and can continue for the duration of force application. Hence, we were able to achieve RCR without any root resorption efficiently in a shorter duration of time using distraction technique.

\section{CONCLUSION}

Distraction osteogenesis for rapid orthodontic tooth movement is an effective technique. Acceleration of canine retraction using the abovementioned techniques has the definitive advantages: Anchorage teeth can withstand the retraction forces with no anchorage loss and without clinical or radiographic evidence of complications such as root fracture, root resorption, ankylosis, periodontal problems, and soft tissue dehiscence. The technique reduces orthodontic treatment duration by 6-9 months in patients who need extraction, with no need for extraoral or intraoral anchorage devices and with no unfavorable short-term effects in the periodontal tissues and surrounding structures.

\section{REFERENCES}

1. Sukurica Y, Karaman A, Gürel HG, Dolanmaz D. Rapid canine distalization through segmental alveolar distraction osteogenesis. Angle Orthod 2007;77:226-36.

2. Codivilla A. On the means of lengthening, in the lower limbs, the muscles and tissues which are shortened through deformity 1904. Clin Orthop Relat Res 1994;301:4-9.

3. Ilizarov GA. The principles of the ilizarov method. Bull Hosp Jt Dis Orthop Inst 1988;48:1-11.

4. Ilizarov GA. The tension-stress effect on the genesis and growth of tissues. Part I. The influence of stability of fixation and soft-tissue preservation. Clin Orthop Relat Res
1989;236:249-81.

5. Ilizarov GA. The tension-stress effect on the genesis and growth of tissues: Part II. The influence of the rate and frequency of distraction. Clin Orthop Relat Res 1989;239:263-85.

6. Ilizarov GA. Clinical application of the tension-stress effect for limb lengthening. Clin Orthop Relat Res 1990;250:8-26.

7. McCarthy JG, Schreiber J, Karp N, Thorne CH, Grayson BH. Lengthening the human mandible by gradual distraction. Plast Reconstr Surg 1992;89:1-8.

8. McCarthy JG. The role of distraction osteogenesis in the reconstruction of the mandible in unilateral craniofacial microsomia. Clin Plast Surg 1994;21:625-31.

9. McCarthy JG, Stelnicki EJ, Mehrara BJ, Longaker MT. Distraction osteogenesis of the craniofacial skeleton. Plast Reconstr Surg 2001;107:1812-27.

10. Liou EJ, Huang CS. Rapid canine retraction through distraction of the periodontal ligament. Am J Orthod Dentofacial Orthop 1998;114:372-82.

11. I eri H, Ki ni ci R, Bzizi N, Tüz H. Rapid canine retraction and orthodontic treatment with dentoalveolar distraction osteogenesis. Am J Orthod Dentofacial Orthop 2005;127:533-41.

12. Reitan K. Clinical and histologic observations on tooth movement during and after orthodontic treatment. Am J Orthod 1967;53:721-45.

13. Rygh P. Elimination of hyalinized periodontal tissues associated with orthodontic tooth movement. Scand J Dent Res 1974;82:57-73.

14. Pilon JJ, Kuijpers-Jagtman AM, Maltha JC. Magnitude of orthodontic forces and rate of bodily tooth movement. An experimental study. Am J Orthod Dentofacial Orthop 1996;110:16-23.

15. Reitan K. Initial tissue behavior during apical root resorption. Angle Orthod 1974;44:68-82.

16. Reitan K. Biomechanical principles and reactions. In Graber TM, Swain BF, editors. Current Principles and Techniques. Saint Louis: C.V. Mosby; 1985. P. 108-23.

17. Reitan K. Tissue behavior during orthodontic tooth movement. Am J Orthod 1960;46:881-900. 